\title{
Entangled with Traffic Signs: An Ethnographic Account of Internationals Driving in Mainland China
}

\author{
Dai Hui \\ Department of English, Faculty of Foreign Languages, Guangdong University of Technology, Guangzhou, China \\ Email address: \\ daihui@gdut.edu.cn \\ To cite this article: \\ Dai Hui. Entangled with Traffic Signs: An Ethnographic Account of Internationals Driving in Mainland China. International Journal of \\ Literature and Arts. Special Issue: Humanity and Science: China's Intercultural Communication with the Outside World in the New Era. \\ Vol. 8, No. 3, 2020, pp. 169-176. doi: 10.11648/j.ijla.20200803.20
}

Received: February 27, 2020; Accepted: March 30, 2020; Published: April 23, 2020

\begin{abstract}
This study investigates the difficulty of internationals' driving in non-English Chinese environment by reporting on their encounters and reflections in the traffic contexts. The study adopts a geosemiotic perspective and utilizes an ethnography to study foreigners' usage of traffic signage in Guangzhou, a major and hub city of Mainland China. It collects the verbal and written data of non-Chinese drivers and Chinese traffic authorities as well as scrutinizing some government standards and news reports on this issue. The study finds that foreign sign users are enculturated to an indigenous Chinese driving practice by assimilating into the local "Chinese-only" driving environment. The empirical findings suggest that traffic signs as one form of discourses in place are keyed to culture contextedness for their comprehension and usage by their users as another form of discourses in action. It is therefore argued that although China with its high-end signing system has stepped into a globalized world, the signing system may be less convenient for foreign drivers to mobilize. The contribution of this research rests with two respects: first, it may appeal to more geosemiotic review of signs in the concrete world; second, this study may be helpful in its attempts to make transportation engineers, urban planners, and law enforcers recognize the importance of Romanized versions of traffic signs addressing foreign drivers in Mainland China.
\end{abstract}

Keywords: Traffic Signs, Foreign Drivers, Social Action

\section{Introduction}

In the past two decades, no country like China has achieved so much in exerting its national influence across the world, e.g. "The Shanghai Cooperation Organisation" (2001) and "The Belt and Road Initiative" (2013) etc. This rising tide of economic and cultural connections has ushered in a boom of international visitors, travelers, and sojourners, making it possible for them to mobilize between the cities of China. About 48 million of internationals visited China in 2018 yet no official data of eligible foreign drivers were found in the same time scale. Regarding the territory of the nation and commuting need of this group, those potential drivers should be many. Yet research on the intercultural roles of traffic signs and their interaction with international drivers remains scarce.

As pivotal navigating devices, traffic signage is well investigated. In ergonomics, artificial intelligence has been utilized to resemble and substitute the mechanism of human minds $[1,2,3]$. Research efforts also target at the optimization of signing information systems [4,5]. The comprehensibility of drivers is addressed by scientists [6-8]. Overall, an efficient, legible and recognizable system as well as readership difference are being sufficiently studied. However, little attention has been given to the real-life interaction between traffic signage and their users. Some researchers suggest that traffic signage involves a trio network of human beings, the vehicle and the travelway and urge the study of the interrelationships among these components in order to determine the effects they have on each other [9].

As a burgeoning country geared to high mobility, China has long recognized the importance of semiotic uniformity and user/foreigner-friendliness in traffic. First, several overhauls regarding the official manual Road Traffic Signs and Markings $(1986,1999,2009)$ have been made to improve this infrastructure. Second, the signing system has undergone some Romanization (the display of Latin letters in public) to cater to the needs of domestic non-Chinese drivers. Yet the process is rendered painful both because of the linguistic 
policy and reality of a monolingual community and because of the indigenous transcribing conundrum of Chinese place names [10-12].

Overall, research on traffic signs from natural science is plentiful but very few studies have addressed traffic signs as a communicative system bridging the travelway and (foreign) travelers. Therefore, this study serves as a realist account of driving encounters of internationals as a minor group of commuters in China.

In this paper, I adopt a geosemiotic perspective and choose the social actions of international drivers as a way to investigate the communication between traffic linguistic landscape and their foreign users. The travelers, traffic authorities and traffic signs in Guangzhou are investigated as the hub city is held representative for its data and large traffic volume. Verbal and written data of non-Chinese drivers and Chinese traffic authorities are collected and some government standards and various news reports on this issue are scrutinized.

\section{Background}

\subsection{Traffic Signs and Their Interactive Role in Traffic}

Traffic signs refer to the traffic control devices by using color, shapes, characters and graphics to inform drivers of certain information [13]. Despite slight verbal nuances in their definitions across the world, traffic signs are relatively uniform in their legibility, brevity and unambiguity of messages.

Traditionally, traffic signs have been approached from traffic engineering with three major perspectives. The AI tradition is overwhelmingly featured in traffic signs recognition (TSR) for the enhancement of a model to detect traffic signs for humans. Information filter, visual attention enhancement, image representation and discrimination, and extreme leaning mechanism all propel the outperformance of TSR over humans $[1-3,14]$. Yet this technology is susceptible to environmental factors and are beset by some technical constraints [15]. It can neither decode some complex contexts nor prescribe amorphous driving behaviors. Thus, researchers urge engineers to know how humans function in order to determine the signing effects [9].

The sign-system branch targets at the enhancement of legibility of traffic linguistics and semiotics. Information type, information volume and information complexity are being assessed $[4,5,16]$. But signs are often found not satisfying the drivers' needs, because the signing system may not achieve its intended functions $[17,18]$. Despite all its intentions to improve the functionality of traffic signage, some questions about whether signs are correctly written, friendly inscribed, properly located and reasonably utilized still await answers.

Human difference (age, gender, race and experience) in information processing is investigated [6-8, 19, 20]. Since driving as a demanding task requests a driver to catch a sign, to draw inferences, and to decide on an action, it is necessary to study the "perceptual and attentional and inference processes as well as their interactions, to understand the drivers' behavior in the traffic environment and to design effective road-safety strategies" [8].

The research literature above reveals the de facto of traffic signs as an interactive semiotic system. In short, to make inroads to traffic signage, the usages of its audience shall be understood.

\subsection{Monolingualism in China}

This section introduces the linguistic policy of China, the two official Romanizing standards in a duel and the linguistic reality practiced by the underneath parties. It serves as an explanation to the indigenous and acquired difficulties of an eligible Romanizing scheme for internationals.

There has been a historical debate over Romanizing issues of public signs in China. In the 1950s, Pinyin became the official Roman spelling scheme for Chinese characters. The issue has been much attributed to the political significance that helps maintain the image of national sovereignty ${ }^{1}$, as Pinyin is supposed to represent a new communist China, where Chinese as the only official language is mandated. Yet the display of Pinyin on signs has also been criticized either for its incomprehensibility to foreigners or for its depletion of cultural sources of place names [21].

The non-functionality of Pinyin in traffic engendered a quarrel between two ministries of China. Ministry of Civil Affairs, with its authority in naming roads nationwide, adopts the Pinyin policy in all pedestrian signs and prescribes it as the only legal approach to Romanization of traffic signs. For example, "Dong-Feng-Dong-Lu" is translated into "Dongfeng Donglu" by this standard. But due to its apparent deficiency in geographic connotations, the standard has not been received by the Ministry of Public Security in charge of transportation and traffic signs (for drivers). The ministry has stipulated their own rule for place names. For example, "Dong-Feng-Dong-Lu" is translated into "Dongfeng Road (i.e. Lu) East (i.e. Dong)". As a result, the display of two Romanized versions for one place in Chinese is evident. For the lack of a national code, many provinces and municipalities have worked out their own regional norms of translating strategies for all public signage.

In academia, thoughts on displaying Roman letters in traffic are divergent: one group believes in Pinyin as an ideal tradition $[11,12]$; and the other group support standardization of traffic signage, be it in English or Pinyin [22, 23]. It is found that the Chinese place names encounter a chaos during this conversion [24].

Given the various ideas by authorities and sophistication in the cultural geography and Chinese language, this research cannot decide on one stoke for this linguistic conundrum. This study intends to give an authentic account of the ways Romanized traffic signs are used and felt. Therefore, believing in the validity of geosemiotics, an ethnography is undertaken

\footnotetext{
1 See the website of Ministry of Civil Affairs, People's Republic of China, http://dms.mca.gov.cn/article/flfg/xzfg/201407/20140700667869.shtml, Retrieved 09/08/2014.
} 
aiming to find out how 'lettered' traffic signs and their users communicate with each other.

\section{Methodology}

\subsection{Theoretical Framework}

Geosemiotics is defined as the study of the social meaning of the material placement of signs in the world [25]. Figure 1 is the prototype schema of geosemiotic systems by linking the theories of interaction order [26], visual semiotics [27], and place semiotics [25]. Social action of sign users is the kernel of these three elements. In short, each element contributes its meaningful part to the overall meaning of signs in the world. And the social action lies at the center of interaction between signs and their audience, manifesting its center role in a typical round of sign-user communication. Since social action depends on both an awareness of the semiotic aggregates and an awareness of these aggregates working together through interdiscursive dialogicality to form composite meaning, human action is thus a process of selection among signs.

Drawing on the theory, this study investigates the foreign drivers' actions in traffic. Two questions are proposed: 1 . What difficulties do international drivers encounter while driving in Guangzhou? 2. What modes of behaviours do internationals adopt in the process?

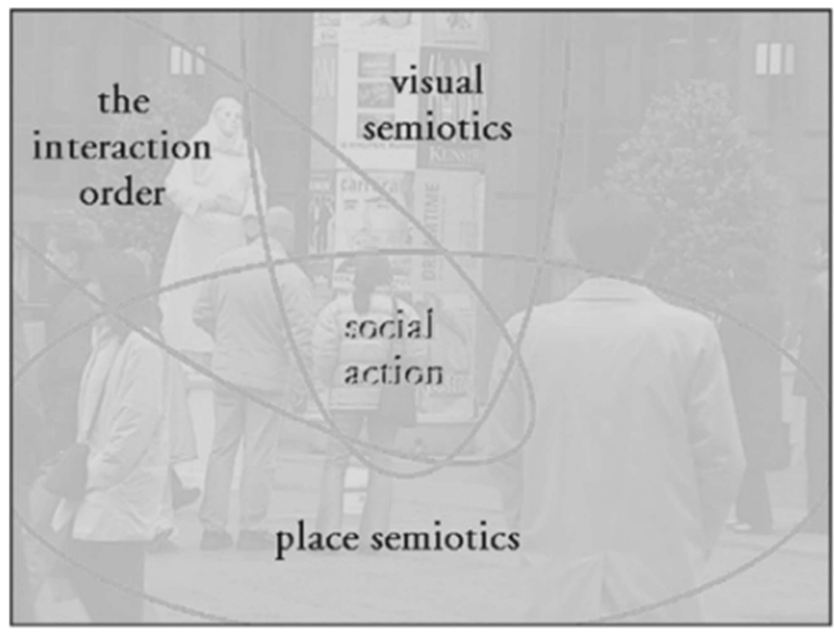

Figure 1. Geosemiotics, Scollon \& Scollon, 2003: 10.

\subsection{Data Collection}

In this study, detailed and open-ended interviews with sample users based on their professions, driving experiences and nationalities were conducted to construct a database. In addition, news reports from websites and newspapers were presented for an overall picture of such usage and interaction in everyday life.

The interviewees were all domiciled in Guangzhou, one hub of Guangdong-Hong Kong-Macao Greater Bay Area initiated in 2017 for the construction of world-level metropolis aggregates. Being a well-established leading city of China, the city's traffic signage is much standardized, especially for its Romanized transcription [28].
This research interviewed more than 20 foreign drivers in Guangzhou, among which Pete, Leo, May, Mon were the typical respondents with their unique perspectives. Also, an Australian traffic expert's (Patrick) thoughts on foreigners' driving in China was quoted. And one prestigious traffic engineer in Guangzhou Laowu was interviewed and provided his trenchant comments. Besides, Cheng and Ren as the veteran drivers with long-term intercultural experiences provided evidence for the difficulties of foreign drivers in China.

\section{Findings}

This section reports the findings in the ethnography of international drivers, on their usages and feelings about their interaction with the Chinese traffic signing system.

(1) The zero codes for non-Chinese drivers

Generally, foreign drivers in China perceive some lack of information when traveling, despite a public awareness of the growth of English display. They may easily notice that many signs are bi-orthographically coded, exuding an international taste and addressing 'Laowai'. The presence of English is seen as a consensus towards globalization and thus instigates some research [29, 30]. However, this trend is accompanied with two facts: overriding monolingualism in traffic information and complaints from foreigners concerning public signs. The absence of English in traffic to address non-Chinese seems to impede China's movement towards internationalization owing to its policy legitimizing Chinese as the only official language for decades. And Backhaus renders such country as still by and large a monolingual country not only ideological but also in practice, despite its linguistic heterogeneity on the rise.

China seems to tally with such description referring to its own code preference, because MCA decreed that Chinese was the only official language since 1957 . Therefore, preponderant Chinese in public solely epitomizes a top-down sociopolitical force. Such speculation is confirmed by many foreign informants in this research. Pete (anonymous, sic passim) is a New Zealander businessman and a driver in China for years. As a frequent international driver (in Australia, the US, Singapore, Hong Kong etc.), he describes his driving and learning experience these years (2010-present) in China.

"The first time I arrived in China was quite chaotic on my part because the traffic signs are in Chinese and many people couldn't speak English well. So I had to ask some of my foreign friends who could speak Chinese to help me in many ways until I met my wife who taught me Mandarin. I think in China, learning Chinese is the best policy. If you want to move around in here, you'd better learn the characters because there are far less English than Chinese. China is a country for Chinese."

In reality, such linguistic impediment does not estrange Pete from many international travelers, especially when they are incapable of speaking the local language. To Pete, the omnipresence of Chinese characters on signage poses a tangible threat in transit. And the situation often worsens when no one around seems to speak English. His hardship was 
initially appeased by his local friends and eventually dissolved by learning Chinese. Such conviction that English letters are still insufficient is echoed by another informant May when she frequently got lost in a labyrinth of monolingual traffic signs by describing them as "not really comprehensible because it's only in Chinese, no English." Finally, she condescended to "just reading signals", the color lights which are universal and foolproof. In the traffic system, the linguistic barrier for foreign drivers results from Chinese as the only authoritative voice in public sphere. Thus, English absent from the national linguistic policy is not much esteemed and only interspersed on streets.

(2) The preference of Pinyin over English

For the traffic signs with two orthographies, a scrutiny of their utility in Roman scripts for their addressees is also necessary. It is surprisingly found that Pinyin in traffic signs wins the approval of foreigners largely.

Despite the facts that public signs are mostly inscribed in Chinese only, English is gaining its steady presence in China, coping with the rising demands of foreign travelers and consolidating the image of a "New China" geared to global integration. However, this bi-orthography is "not representative of the linguistic repertoire of ethnolinguistic diversity but rather of those linguistic resources that...institutions make use of in the public sphere" [31].

In China, bi-orthographic signage such as place names and road names also seem to nurture an ideological battleground between English and Pinyin. The anecdote starts with the national manual on traffic signs RTSM stating that "Chinese characters and other characters can coexist when necessary" [13]. Yet it makes no reference to any converting rules for Chinese signage and brings about two outcomes: (1) the protracted dispute of two government sectors (MCA for Pinyin and MPS for English), both of which empower themselves with their own transcribing rationale and application; (2) the solo practice of linguistic conversion by each municipal government to make public signs 'culturally-distinctive'.

Largely speaking, the bi-orthography in China is recognized as "generally good" by many foreign informants. Yet owing to some regional toponym differences (e.g. hutong in Beijing and nong in Shanghai), the comprehensive uniformity on converting geographic names across the country seems futile. Leo is an American and has been driving in China since 2003. And his Chinese driver's license looks identical to those of the natives. The registrar police happened to give him a requisite and catchy Chinese name for his driver's license-Li Yiwen). As a non-Chinese driver in Guangzhou, he welcomes a transition of traffic signs from Chinese-only to two orthographies these years. And he reflects on his interaction with traffic signs,

"By the time I arrived in Guangzhou (in 2003), direction signs were not in English or Pinyin. Now many of those signs are in Pinyin, making it a lot easier to go around. In larger cities the use of English letters to display destination is good. In cities like Guangzhou this goes down to the individual urban street level. Translations of signs are few and far between and largely useless."

May confirms this inclination to use Pinyin by asking her Chinese students to spell her destination in letters in advance. She makes a point of the translating problem in her own case,

"[When] I am speaking to a Chinese, I say Tian-He-Ke-Yun-Zhan because that is more likely for me to get help from a Chinese passerby. I know Tian-He-Ke-Yun-Zhan actually means Tianhe Bus Station. You see there is no one using that, even among us, even among foreigners. Besides, sometimes the English translation is a little funny because the grammar is all wrong."

It seems from the perspective of a driver, the choice of Pinyin is grounded on certain rational consideration, for effective communication with locals. In effect, the usage of Pinyin to refer to destination indicates foreigners' practices to ask around in traffic by the Chinese pronunciation, rather than a belief to really get somewhere by means of signs only.

Despite the Roman-script issues examined above, there arises another dilemma for Chinese to be translated into English-verbosity, generated from C-E transformation because of the increasing amount in byte and space. Mon expresses his concern about the translation of signs,

"I don't know if it's Asian or Chinese but they tend to be wordy. They use a lot of words. I mean sometimes I just need the symbols. So instead of just saying a sign that goes left and says 'the number 458 Hospital of People's Liberation Army. So long! What's that? I guess I have to ignore them."

Mon's need for symbols may sound confusing but reveals the profound coding difference between an English traffic signing system and a Chinese one. It may result from a literal understanding of the meaning of place names and their functions. For example, in the British signing system, the hospital is only abstracted as one symbol-a big " $\mathrm{H}$ " representing an adjacent medical service, for drivers' emergent use. But the full name of a nearby hospital facility means a lot more in China: it has to index its seekers about its title and location; and it has to index the visitors about its quality service and important role seen from an official sign filtered through a "rigorous grading system" [13].

The display of English in public signage epitomizes the movement of an ancient monolingual society towards an English-dominated world. But the accounts reveal that English is underutilized for "funny", "incomprehensible", "wordy" translations. The use of Pinyin instead of English implies that the English landscape may be "symbolic construction of the public space" [31].

A globalized China seems to require foreign visitors to prepare for their itinerary in order to evade a deflected course. Their traveling in the country demands 'backup plans' (spell Pinyin) or 'backup plans for backup plans' (spell both Pinyin and characters) which ultimately target at the Chinese helpers. These examples prove their common mode of traveling - to ask passers-by, to call some acquaintances, to display Pinyin and characters all together as double check. Surprisingly, most foreigners seem to be acculturated with quite an adaptability towards the Chinese way of mobilizing and socializing-by asking people, instead of relying on signage. Inquiring the 
locals for information is discussed in Part (5).

(3) Ephemeral roads and road names

An issue in comprehending the traffic information both for Chinese and for internationals is discussed, i.e. road naming and renaming. This rule of polysemy has its far-reaching influence on geographic names. Presumably, foreigners would find it difficult to adapt themselves to the alterations of road names, a frequent social practice indigenous to the Chinese traffic contexts.

Yangcheng Evening reported in 2011 that there appeared 1600 new road names before Asian Games in 2012. And Guangzhou was reported ranking first nationwide with its biggest number of road names. Among this tide, one typical example of Project HEMC in Guangzhou, a 34.5 square-kilometer island dwelling ten major universities, is worth mentioning.

This island was secluded and nameless before it was constructed as a university town. The project was planned and then hastily started in January 2003 and accomplished and put to service in September 2004. Roads obtain their names by the road networks consisting of three ring roads (inner, middle and outer) and many yoke roads partitioned by four directions (north, south, west, east). and five numbers (1-5), e.g. Nan-Yi-Lu literally means South One Road.

The traffic engineer Laowu, a witness of this road naming project, regards such practice as "a little simplistic" because the road naming and renaming "may be the say-so" of nomenclators who claim that this method is learned from "the west". When about 250,000 students kept thronging in annually since 2004 , more and more lost drivers emerged and they criticized the perplexing road names, claiming their difficulty in distinguishing between directions and numbers in road names. Thus, the administrators began their revision project in 2013.

In 2013, the names on yoke roads are changed, from the "direction+number" pattern to a "verb+object" pattern (e.g. Shang-Fa-Jie, Upholding-Law-Street and Qiu-Zhen-Jie, Pursuing-Truth-Street). Because most directive infrastructure need to be renovated, the renewal last for about three years (2013-2016). As a result, two names for one road coexisted for several years, making it hard for drivers to mobilize.

Foreign drivers on this island do not show much sensitivity to the revised names. The road renaming practice feels somewhat intangible to them as May shares her feelings.

"Honestly, I didn't even notice the change. Driving in China, I don't memorize those names. They are in Chinese and too complex. The translations are always changeable. Flip-flops are quite common in the country. Living here, you sure need to make adjustments."

Some researcher regards changes in place names as a loss of cultural assets for the fact that China has sustained this changing tradition for many dynasties [32]. With the long and divided history of the country, geographic names seem to become a representation of political will, namely a hectic willingness to remake nature. For people with the power to effect the changes, the geographic civilization is more of an ideological concept than a cultural one.
(4) Unspecific frame of reference and its influence on drivers

It is believed that the Chinese culture tends to code messages implicitly and ambiguously, which is well substantiated by traffic signage. The 15 -year veteran driver Cheng explains the facilitating role of traffic signs in direction finding and ascribes such notion to the unspecific grid or reference of the given system. He summarizes,

"Your own bad luck if going astray by following signs. Driving to a new place requires a series of strategies. First set GPS or your cell on navigation. Then be wary of those places. Sort out the major places and then resort to those signs. One thing is that signs are somehow used as a proofreading mechanism for your choice. I am not used to relying on signs believing that they can lead me to wherever I want. For example, a sign tells that it leads to Guangzhou Avenue. But the avenue can stretch like twenty kilometers, and it simply won't and can't tell you which particular part the sign points to, whether it's in the middle, south or north section. Our grid of reference is unspecific, thus dwindles signs' usage."

It seems that from the perspective of experienced drivers the undervaluation of signage is reasonable for its secondary role for an effective journey. He suggests certain prior knowledge for drivers to travel in China. Clearly, driving in China involves a set of preparatory activities: finding a navigating device, then sorting out major places, and then specifying the road section of a desired place. It is the last step to match "Column A with Column B".

The experienced driver expresses a distrust of traffic signs because the Chinese navigating information lacks concretion. Thus, transporting to a new place does not involve reading traffic signs impromptu because they merely act as a "proofreading mechanism", a tool to help correct the human/navigation mistakes.

Cheng's belief is triangulated by Ren's own intercultural driving experiences. Ren is a Chinese driver who studied and drove in the UK. He attributes the crucial difference in designing signs in both countries to the forethought for diverse users. And Ren summarizes his findings in user-friendliness of signs between the two countries.

"Our traffic signs point to a road, a broad-scoped direction, like Huan-Shi-Xi-Lu (Huanshi Road West). Instead, the British signs target at specific places, like Westminster. They seldom point to roads [except for major routes]. It is just under the circumstance that I am not familiar with a road and its neighborhood that I perceive a need for traffic information. But unfortunately, traffic signs in China only show you which road to take so vaguely that you must remember which way to go. Conversely in the UK, the signs are constantly reminding you of which way to go, either in the front, or on the ground, or else, treating you as kids or even idiots."

A common notion of the signing system in China is that signs are built on the drivers' certain familiarity with their routes. Ren goes on evaluating this practice in China:

"The assumption is that you must have studied your itinerary in advance. You should know where your destination is located - on which specific road, to which direction, etc. 
You shall figure it out by yourself beforehand and come back here to meet the signs. Overall, Chinese signs are less human-oriented."

Ren complains of this "knowing first" assumption for traffic regulations and signs, a hidden dimension underneath the explicitly-coded traffic rules - the hospitality floating on the surface and hostility underneath.

(5) A dă-tīng (inquiry) culture

The meaning of traffic signs may result from/in a particular social practice on the roads in China-asking local people for traffic information. In the Chinese context, dă-tīng means to verbally inquire for reliable knowledge of something, some people or place not apparent or available. Two elements lay foundations for the dă-tīng culture: the colloquial competence of a local language; and the social connections of a given culture. That is, for something less familiar, one has to put himself/herself in verbal communication with people instead of dehumanized machines or signage. This is the high-context way of socialization and information access, in which "great and deep involvement of the participants with each other" is advocated [33].

The dă-tīng practice seems to be a shared behavioral pattern of all foreign and domestic informants in this study. They all express some reasonable faith in learning Chinese for long-term stay in China in that "there are a lot of Chinese if we get lost we can ask them" (Mon) and "I ask some of my foreign friends who could speak Chinese for help" (Pete) or "I really ask my students and police" (May). Plausibly, the competence of the Chinese language constitutes a touchstone in order to interact in the Chinese-only situations.

It cannot be denied that modern transportation in China is now much facilitated by many navigational systems such as Baidu Map and Amap. Yet due to the facts of complex natural geography, stereoscopic roadway constructs and monolingual linguistic landscape, inquiries are likely to persist.

(6) Lack of traffic etiquettes

In order to discover more about the social action of drivers, it is necessary to comprehend their "habitus" in a context-dependent sociocultural situation, denoting "the accumulated experience and knowledge both conscious and unconscious of a social actor which is theorized as the primary source of human action [25].

The drivers' habitus may have far-reaching influence on their social behaviors in traffic and vice versa. It is found that that all foreigners verbalize their anxiety and fear about the ways other drivers behave, a collateral evidence of the implementation, transaction and enforcement of traffic signage and its peripheries. Therefore, in what follows, civil opinions about traffic signs, other control devices, and traffic per se are revealed by foreigners.

Where there is a will, there is a way

Here are some accounts of foreigner drivers in China, regarding the driving propensity of Chinese people. Patrick is an Australian traffic expert on road safety. In 2007, he became one of the licensed drivers in China. He describes such a goose-bump driving situation that may shed light on how a Chinese traffic scenario unfolds.
"There are three marked lanes on the expressway, however, there appears to be four lanes of actual traffic. Many drivers weave in and out of lanes at will and even create their own lane, between lanes. Nobody uses indicators and beeping horns are constant. Without a warning, another car cuts in front, and your heart plummets again."

Traditionally, Lane markings are "supported by laws, ordinances, or regulations" (Sect. 1A. 08), and "command respect from road users" (Sect. 1A. 01) as an ancillary traffic signing system [34]. Therefore, the authority of each lane shall be strictly obeyed by drivers.

However, it is often found that in China the distinct three lanes can be practiced in four and drivers are free to create their lane regardless of the compulsory control. This may suggest the traffic laws are not respected. And the idea is shared by all foreign informants in this research who report on a disorder in driving. And Pete shares his experience,

"The law in China is generally not bad. The problem is nobody adheres to it. At junctions with traffic lights it would be hoped that vehicles would stop for the red light and go only on the green light. However, this is not the case, with larger vehicles still streaming across the junction seconds after the light has turned red, and smaller vehicles and pedestrians ignoring the lights altogether and trying to get across the junction any way they can. Go on the green light, but be careful."

Since 2004, China has implemented stricter laws and regulations (often with more strengthened enforcement cameras) for drivers, prescribing a package of rules for occurrences and behaviors on the road. Yet such regulations rest on several factors to effect (e.g. drivers, cars, law enforcers, conditions and regulations, etc.). Since "nobody adheres to it", the law may remain stipulations on the book.

As a nation with more than two thousand years of history and culture, China has achieved a fame of both being agricultural and mysterious. Since the Open-Door Policy in 1978, China has been speeding ahead towards becoming an industrial country. However, this innovative breeze along with reform of government and enterprises, for all their efforts and achievements in economy, in science, and in infrastructure and projects, seem to be ineffective to wash off the conventional mentality and traffic ethos that have sustained for millennia.

\section{Traffic laws biasing lawful drivers: might is right}

Since traffic laws in China are taken less seriously by civilians, there is reason to believe that peace-loving and law-abiding drivers may not be paid for their observance of the traffic laws. Pete shares his deliberations on the roadway justice,

"The traffic law has a bias towards reducing collisions and being polite, which actually states that law-abiding citizens should give way politely to those breaking the law and driving impolitely. This is actually no different in the West, except that there are more vehicles with a greater range of sizes and speeds, with a far greater percentage breaking the law and being driven selfishly."

Then he quotes a specific case, 
"Roundabouts are not explained in Chinese driving test material, apart from that drivers on the roundabout should give way to vehicles entering without priority. So roundabouts have the ridiculously inefficient situation where only the vehicle that gets there first has priority and then only if it is bigger or traveling faster or with more "purpose". Take care. Keeping to the outside of the roundabout is recommended."

Also, the "hard-look" approach adopted by law enforcers in China may bring about the topic of a forgiving road believing that roads should be designed with the thought that people will make a mistake. There is this common case when drivers having had some traffic misconduct and were stopped by traffic police issuing a ticket and claiming that a "law" is around and shall not be transgressed. As a foreign driver in China, Leo describes his interaction with traffic signs and a law enforcer,

"My first bad experience with a traffic sign was ridiculous. I took a right turn on a street only allowed for buses. The cop stopped me and explained my infraction. I tried to explain to him that the sign was in Chinese and I couldn't read it. He still gave me a ticket. I thought that was unfair. The cop could have let me go with a warning."

In the Chinese society, drivers are largely held accountable for themselves when they choose to mobilize. And traffic signage and law enforcers provide no further guarantee and carry less liability for signs' legibility. Regular drivers may be preprogrammed with certain knowledge while driving on the Chinese roads. But for foreigners who have no such information access, some price is to be paid.

\section{Conclusion}

Given the large number of passengers in and out of China, there should be many internationals driving in the country. There may be even more foreigners willing to drive on this land. Yet little research has been found on them. Thus, their needs and feelings for a user-friendly signing system are enormously ignored. By the ethnographic account of foreign drivers' experiences, usages and feelings, this research reports on the interaction of traffic signs and their foreign users.

The analysis yields the following findings. First, there are no proper traffic messages addressing English speakers, indicating that the traffic linguistic landscape is dominated by Chinese and Romanized letters play a minor role in traffic life. Second, two conflicting voices Pinyin and English respectively make their own inroads to the public spheres. This brings about the chaos of the bi-orthographic situation in the Chinese traffic landscape. Third, the rising emergence of geographic constructions (highways, expressways, streets, etc.) and their inconstant names make their navigating tasks an unsettled trouble. Fourth, the signing mode of roads rather than places requests its users to study the routes beforehand in order to establish certain familiarity with their own itineraries, raising the threshold competence for internationals driving in China. Five, the indigenous navigating strategy to ask around regardless of signage in China is pervasive and indicates a traffic world built upon acquaintanceship. Last, the missing link between the ancient Chinese civilization and the competitive traffic world is apparent, adding to the intercultural difficulties of international drivers. Overall, it is presumable that foreigners in China are forced to adopt the locals' mode of traveling by interacting with Chinese signs (reading Chinese and following its pronunciation) and Chinese people (by asking around).

The study does not intend to argue against the utility of traffic signs for foreigners as the national network transports the world's largest population and does facilitate many internationals driving in China. Rather, by providing some authentic accounts of usages and feelings of foreign drivers, the study may help identify some possible improvements for this important infrastructure in order to serve its marginalized users.

\section{References}

[1] Sun, Z., Wang, H., Lau, W., Seet, G., and Wang, D. (2014). Application of BW-ELM model on traffic sign recognition. Neurocomputing, 128: 153-159.

[2] Gao, X. W., Podladchikova, L., Hong K., and Shevtsova, N. (2006). Recognition of traffic signs based on their color and shape features extracted using human vision models. Journal of Visual Communication \& Image Representation, 17: 675-685.

[3] Ruta, A., Li, Y., and Liu, X. (2010). Real-time traffic sign recognition from video by class-specific discriminative features. Pattern Recognition, 43: 416-430.

[4] Shinar, D. and Vogelzang, M. (2013). Comprehension of traffic signs with symbolic versus text displays. Transportation Research Part F, 18: 72-82.

[5] Liu, Y. (2005). A simulated study on the effects of information volume on traffic signs, viewing strategies and sign familiarity upon driver's visual search performance. International Journal of Industrial Ergonomics, 35: 1147-1158.

[6] Kirmizioglu, E., and Tuydes-Yaman, H. (2012). Comprehensibility of traffic signs among urban drivers in Turkey. Accident Analysis and Prevention, 45: 131-141.

[7] Ou, Y. K and Liu, Y. C. (2012). Effects on sign design features and training on comprehension of traffic signs in Taiwanese and Vietnamese user groups. International Journal of Industrial Ergonomics, 42: 1-7.

[8] Roca, J., Castro, C, Bueno, M., and Moreno-Rios, S. (2012). A driving-emulation task to study the integration of goals with obligatory and prohibitory traffic signs. Applied Ergonomics, 43 (1): 81-88.

[9] Hoel, L. A., Garber, N. J., and Sadek, A. W. (2011). Transportation Infrastructure Engineering: A Multimodal Integration. Stamford: Cengage Learning.

[10] Xu, Q. and Liu, L. (2012). Promoting the national uniform Romanization and preserving the cultural diversity. China Municipal Affairs, 5: 40-41.

[11] Guo, J. (2003). Romanization of road names. Chinese Translators Journal, 5: 83-84. 
[12] Guo, J. (2007). National standards and international norms of the alphabetical writing system for road signs. Chinese Translators Journal, 5: 68-71.

[13] Road Traffic Signs and Markings [RTSM]. (2009). Beijing: Standards Press of China.

[14] Guo, H., Wang, X., Zhong, Y, and Lu, P. (2011). Traffic signs recognition based on visual attention mechanism. The Journal of China Universities of Posts and Telecommunications, 18: 12-16.

[15] Jimenez P. G., Bascon, S. M., Moreno, H. G., Arroyo, S. L., and Ferreras, F. L. (2008). Traffic sign shape classification and localization based on the normalized FFT of the signature of blobs and 2D homographies. Signal Process, 88: 2943-2955.

[16] Liu, B., Wang, Z., Song, G, and Wu, G. (2010). Cognitive processing of traffic signs in immersive virtual reality environment: An ERP study. Neuroscience Letters, 485: 43-48.

[17] Summala, H. and Hietamäki, J. (1984). Drivers' immediate responses to traffic signs. Ergonomics, 27 (2): 205-216.

[18] Fisher, J. (1992). Testing the effect of road traffic signs information value on driver behavior. Human Factors, 34 (2): 231-237.

[19] Ng, A. W. Y. and Chan, A. H. S. (2008). The effects of driver factors and sign design features on the comprehensibility of traffic signs. Journal of Safety Research, 39: 321-328.

[20] Xie, C. and Parker, D. (2002). A social psychological approach to driving violations in two Chinese cities. Transportation Research Part F, 5: 293-308.

[21] Ge, X. (2009). Reflections on the Romanization of Chinese Geographical Names. Journal of PLA University of Foreign Languages, 2: 61-66.

[22] Ge, X. and Ji, Z. (2006). Ways out: English translation of place names. Shanghai Journal of Translators, 3: 57-59.
[23] Ye, Z. (2000). Globalization and translation of standardized language. Chinese Translators Journal, 2: 8-13.

[24] Dai, H. (2015). A Geosemiotic Study of Communication Between Traffic Signs and Their Users in China. PhD Dissertation. Guangdong University of Foreign Studies.

[25] Scollon, R. and Scollon, S. W. (2003). Discourses in Place. London: Routledge.

[26] Goffman, E. (1983). The interaction ritual. American Sociological Review, 48: 1-19.

[27] Kress, G. and van Leeuwen, T. (1996). Reading Images: The Grammar of Visual Design. London: Routledge.

[28] Specification for English Translation of Public Signs [SETPS]. (2008). Guangzhou: Guangzhou Foreign Affairs Office, Geographical Names Office, and Bureau of Standardization.

[29] Heller, M. (2003). Globalization, the new economy and the commodification of language. Journal of Sociolinguistics, 7 (4) 473-492.

[30] Backhaus, P. (2009). Rules and regulations in linguistic landscaping: A comparative perspective. In E. Shohamy and D. Gorter (Eds.) Linguistic Landscape: Expanding the Scenery, 157-172. New York: Routledge.

[31] Ben-Rafael, E., Shohamy, E., Amara, M. H., and Trumper-Hecht, N (2006). linguistic landscape as symbolic construction of the public space. In D. Gorter (Ed.) Linguistic landscape: A New Approach to Multilingualism, 7-30. Clevedon: Multilingual Matters Ltd.

[32] Deng, Q. (2011). Aspects of road renaming and name restoration. China geographical Names, 28-30.

[33] Hall, E. T. and Hall, M. R. (1987). Hidden Differences: Doing Business with the Japanese. New York: Anchor Books.

[34] Manual on Uniform Traffic Control Devices [MUTCD]. (2009). Washington, D. C: US Department of Transportation, Federal Highway Administration. 